Portland State University

PDXScholar

8-1-2016

\title{
Production Planning Using Evolving Demand Forecasts in the Automotive Industry
}

\author{
Hakan Yildiz \\ Michigan State University \\ Scott DuHadway \\ Portland State University, duhadway@pdx.edu \\ Ram Narasimhan \\ Michigan State University \\ Sriram Narayanan \\ Michigan State University
}

Follow this and additional works at: https://pdxscholar.library.pdx.edu/busadmin_fac

Part of the Operations and Supply Chain Management Commons

Let us know how access to this document benefits you.

\section{Citation Details}

Yildiz, H., DuHadway, S., Narasimhan, R., \& Narayanan, S. Production Planning Using Evolving Demand Forecasts in the Automotive Industry.

This Post-Print is brought to you for free and open access. It has been accepted for inclusion in Business Faculty Publications and Presentations by an authorized administrator of PDXScholar. Please contact us if we can make this document more accessible: pdxscholar@pdx.edu. 


\title{
Production Planning Using Evolving Demand Forecasts in the Automotive Industry Hakan Yildiz, Scott DuHadway, Ram Narasimhan and Sriram Narayanan
}

\author{
Department of Supply Chain Management \\ Michigan State University, East Lansing, MI 48864
}

\begin{abstract}
This paper considers an auto parts supplier who receives order release updates from its customers and revises its production plan for future periods on a weekly basis. The inaccuracy of the order releases causes significant costs in the form of premium expedited transportation, production overtime and excess inventory. This setting provides a rich context for studying order release variance because the supply chain has adopted a JIT approach where ideal inventory levels are kept at zero. This leads to a high reliance on order release accuracy in order to manage production quantities. This paper presents an optimization model that extends previous approaches focused on optimizing production plans to the JIT setting. Further, based on real order release information provided to the supplier, two simple adjustment heuristic methods are developed. The performances of these approaches are compared to relying solely on order releases received from the customers. The simple median based adjustment heuristic performs the best of all the approaches. Implications of the analysis are also discussed.
\end{abstract}

Index Terms-Forecasting, forecast evolution, heuristics, linear optimization, production/inventory management, supply chain integration

\section{INTRODUCTION}

One core aspect of effective communication within a supply chain involves the flow of timely and accurate information upwards in a supply chain from the end consumer to the various 
suppliers. In order for the information to be of value, the information needs to be provided early enough so that suppliers can account for adjusting production quantities and order quantities in anticipation of demand changes. The information also needs to accurately represent future quantities so that supply chain partners can appropriately react. When this information is not accurate or timely, negative effects occur within a supply chain including bullwhip [1], higher levels of inventory, expedited shipping costs [2], and over/under production [3].

This study is motivated by a real forecasting/production planning problem faced by a major first tier auto parts supplier (referred to as Parts Inc.) who supplies to automobile manufacturers in the US, Europe, and Asia. One of these automobile manufacturers (referred to as Car Co.) releases weekly preliminary orders (i.e. forecasts) to Parts Inc. These order releases are estimates for actual orders starting from several weeks before the due date. Car Co. can update their order releases anywhere from the current week to forty weeks out. Suppliers of Parts Inc. have long lead times and do not have inventory to adjust to changes in these releases quickly, which results in premium freight costs. Therefore, production planning decisions have to be made carefully by both the Parts Inc. and its suppliers in order to achieve high service levels and lower premium freight costs.

There were two key drivers of Parts Inc.'s inventory and replenishment strategy: having a true JIT system with no safety stock, and meeting fluctuating demand requests from Car Co. The primary problem faced by Parts Inc. was that the order release quantities forecasted by the customer for the same future time period had high levels of fluctuation between different weeks. High variation in order releases led to part shortages and expedited freight shipping costs. Parts Inc. has difficulty in analyzing and relying on the Car Co. order releases over time as an input to production planning efforts. Thus Parts Inc. was in need of a simple approach to analyzing the 
order release update behavior of Car Co., which can be used to alert Parts Inc. and its suppliers of potential changes of future orders and consequently lead to reduced supply chain costs. In addition, such an approach must be fast since there are hundreds of parts to look at each week. Currently this is handled by material managers manually and it is very time consuming. This will provide visibility to manage order release volatility more proactively.

The automotive industry provides a unique and rich context for studying the problem of evolution of order releases and their accuracy because of the specific expectations that the automobile OEMs have of their suppliers. Specifically, many OEMs operate in a JIT environment and do not carry safety stock. Because of that, they expect their suppliers to provide the parts in a timely manner even if that meant that the supplier expedites shipment or rushes production, both of which are expensive.

Although there is effort on the part of the OEMs to work closely with their suppliers, in many ways the industry is still driven by the OEM, which pushes its influence throughout the supply chain. So the suppliers are reliant on information provided by the OEM for forecasting information.

A number of researchers have developed and used optimization techniques to suggest appropriate models for managing order release variance. Graves et al., [5] focuses on adjusting forecast information using changes in order release quantities to have optimal safety stock levels. Huggins and Olsen [6] address a very similar problem by balancing inventory levels with expediting costs in response to managing order release variance. Altintas and Trick [7] suggest a tool to compare different customers based on order release accuracy and bias in order to create a performance analysis of customers. These studies highlight the challenge of utilizing order release information in order to have higher performance in the existence of order release 
variance. In addition, they demonstrate different approaches that have been used to manage order release variance, including inventory, expediting, and customer classification.

Following the previous research, we focus on identifying and evaluating strategies for managing order release variance. Our research diverges in a number of critical ways. First, our research focuses on a manufacturing setting of strict JIT where safety stock is set to zero and the supplier did not carry a buffer. Thus Parts Co. cannot rely on inventory as a buffer for managing order release variance. This requires careful management and optimization of order release variance rather than relying on inventory. Poor production plans and inability to manage order release variance lead to significant costs from both excess inventory, due to overproduction, which disrupts the strategic orientation of the company, and expedited shipping costs when shortages occur. Second, we use actual order release data provided to Parts Inc. by Car Co. and the methods we used do not rely on assumptions of normality, unbiasedness or stationary data, which is not the case in past studies. Third, our research presents both an optimization modeling approach and two heuristics that are then compared against one another. This provides a test of a simple solution that manufacturers can implement compared to the more complex modeling approaches. Both the modeling approach and the heuristic are useful for providing unique and interesting insights about the data.

The optimization method, which we will discuss more fully later on in the paper, acts as an expansion of and a test for the findings in the Graves et al., [5] paper about the value of order release update information for production planning purposes. Using the order release data received by Parts Inc., we model the optimal usage of order release quantity change information in revising the production plan for future periods. The findings in the model suggest that adjustments to order releases provide valuable information for changes in future weeks (i.e. the 
change in order release quantities for period $\mathrm{T}+2$ is relevant for adjusting the production plans for $\mathrm{T}+2$ as well as $\mathrm{T}+1$ and $\mathrm{T}+3$ ).

The heuristic approach is based on the observation that the Car Co. consistently changes its order release quantities for the actual orders in the last minute. The heuristic adjusts the production plan based on a historic adjustment factor and the order release information received. When compared with the optimization method, the heuristic approach provides a more accurate tool for predicting actual quantities of production without relying on the complicated modeling approach. Thus, both approaches are useful in analysis.

\section{LITERATURE REVIEW}

Demand uncertainty is a major supply chain risk faced by managers and can have major impact on production planning, and consequently profitable operations [20]. Because of that, the accuracy of the order releases and the evolution of order releases over time have received significant attention in the literature. There has been emphasis in the literature on dynamic modeling of requirements planning from customer forecasts $[9,10]$. One key approach is the Martingale model of forecast evolution (MMFE) such as that by Heath and Jackson [11]. The other is the dynamic models such as presented by Graves et al. [5], which assumes that updates closer to the production period make the forecasts more accurate. Based on this assumption, they calculate optimal weights for forecast updates within the planning horizon to smoothen production plans. In contrast, Cattani and Hausman [4] investigate why forecast updates are often disappointing. A key result of their study is that there is randomness in forecast data, and updates can frequently result in poor forecast accuracy. They conclude that "many manufacturers and business managers may have unrealistic expectations concerning forecast accuracy improvements as forecasts are updated in the final periods before the demand event." [4; p. 125]. 
Thus, it is unclear how should forecast updates influence the production planning process and whether this depends on the industry characteristics of the firms experiencing this type of a problem.

Others such as Hausman [8] provide a dynamic programming analysis of forecast evolution, which provided a building block for future research on forecast evolutions. Dynamic programming is an effective theoretical framework for analyzing optimal theoretical properties of forecast evolution, but difficult to apply in practice [10]. Simplicity and ease of use is a key aspect of a methodology embraced by practitioners since managers shy away from complex forecasting methods and get stuck with their preferred methods of choice [19]. Many firms may not have capabilities to use complex methods. Moreover, many of the complex methods require the data to satisfy some assumptions before they can be applied, which may make these methods not very useful in practice. Furthermore, firms such as Parts Inc. may not necessarily place equal weight on shortage and overage costs that are natural outcomes of forecasts that are never perfect. However traditional forecasting methods treat both inaccuracies as equivalent. For these reasons, we consider an adaptation and test of the Graves et al. [5] optimization model and compare it with a heuristic technique, which can be easily adopted into practical use.

Forecasting through collaboration has been shown to have impact on supply chain performance. Aviv $[13,14]$ finds that collaboration can lead to superior inventory management performance. Terwiesch et al. [15] finds that forecast sharing can lead to significantly better performance but is hampered by self-interested forecast inflation and variance. Altintas and Trick [7] provide a framework to compare forecast performance of different automobile manufacturers, which divides customers into separate groups based on forecast biases and found that customers were consistent in biased forecast behavior. However, the paper did not offer 
strategies for managing forecast biases. In buyer-seller relationships where suppliers are absolutely required to satisfy the orders fully, due to contractual requirements, keeping safety stock is one of the important means to achieve this. Huggins and Olsen [6] consider the scenario where order quantities are required to be met through expediting when they are unable to be met through inventory. Their research focused on optimal inventory levels when considering the balance between inventory levels and expediting costs. In the context of our study, keeping safety stock is not an option due to the JIT manufacturing environment that both Car Co. and Parts Inc. are in. Thus, revising the production plan in order to manage order release variance according to firm objectives is the main focus of our study. Poor production plans lead to significant costs from both excess inventory, due to overproduction, and expedited shipping costs when shortages occur.

Previous research has also called for additional research on the structure of the demand [6]. This paper is based on analysis of actual order release data in the relationship between Parts Inc. and Car Co. and does not rely on assumptions of normality, unbiasedness or stationary data in its analysis, which is not the case in past studies. Using actual order information allows a test of previous models presented in the literature such as Graves et al. [5].

\section{Methodology}

We first compiled the historical order releases received by Parts Inc. from Car Co. Next, the release data were examined for data irregularities prior to automation. Furthermore, everything needed to be done in a spreadsheet environment so that the managers can make sense of the analysis with their existing capabilities. Once the data was structured and regularized, it was realized that there are several data trends that can be of value. Table I provides sample order release data and the aforementioned data trends. 
In Table I, rows represent the order release received at a certain week. For instance, for week $\mathrm{T}$, which was received on $6 / 23 / 2014$, the actual order quantity for week $\mathrm{T}$ was 82 . The anticipated order quantities for the following weeks are 80, 113, etc. The first data trend is the evolution of the order quantity over time, which is found in the columns in Table I. Although the demand for week T was 82, the previous week, Car Co. anticipated that a demand of 66.

Similarly, the order quantity was 77, 76, and 57 in the order releases sent two, three and four weeks ago, respectively. Depending on the lead time of the part in consideration, Parts Inc. needs

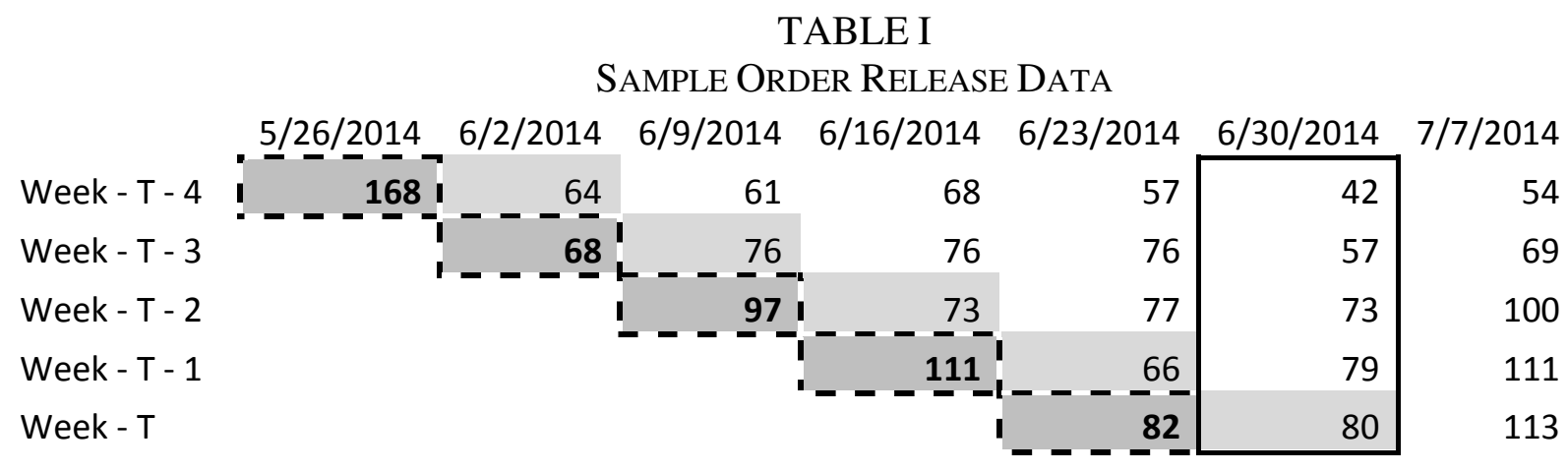

to make a production plan and transmit that information to its suppliers so that demand and production can be matched upstream. For instance, for this part number, the lead time is one week. Thus for week T's production, the last order quantity received was 66. If Parts Inc. relies on this quantity, it would end up with a shortage of 82-66 = 16 units, which needs to be expedited by overtime production and premium freight.

The second data trend is the actual order quantities, which is the highlighted diagonal on the lower left side of Table I. The order quantities vary from week to week, starting at 168 in the week of 5/26/2014 and continuing in the following weeks with $68,97,111$, and 82 . This is the demand information for each week. In addition to the actual demand, each of the other diagonal data represents the week-out order release quantities. For instance, the next diagonal is the 1- 
week out order release quantities.

The third data trend is dependent on the lead time of the part. If the lead time is one week, for instance, then the difference between the actual order and the 1-week out order release quantity for the same week is the relative change in order quantity. In Table I, this data is $68-64=4$ at week T-3, 97-76 = 21 for week T-2, etc. By dividing these by the 1-week out order release quantity, we find the percentage change. For example for week T-3, the change is $21 / 76=$ 27.6\%. A sample data set with 40 weeks order releases for a specific part is given in Appendix 1, where the data is transformed for confidentiality reasons. Figure 1 partially illustrates all of three data trends for a specific part. The solid line at the top is the actual demands for each week. The remaining three dashed lines are the corresponding previous order release quantities.

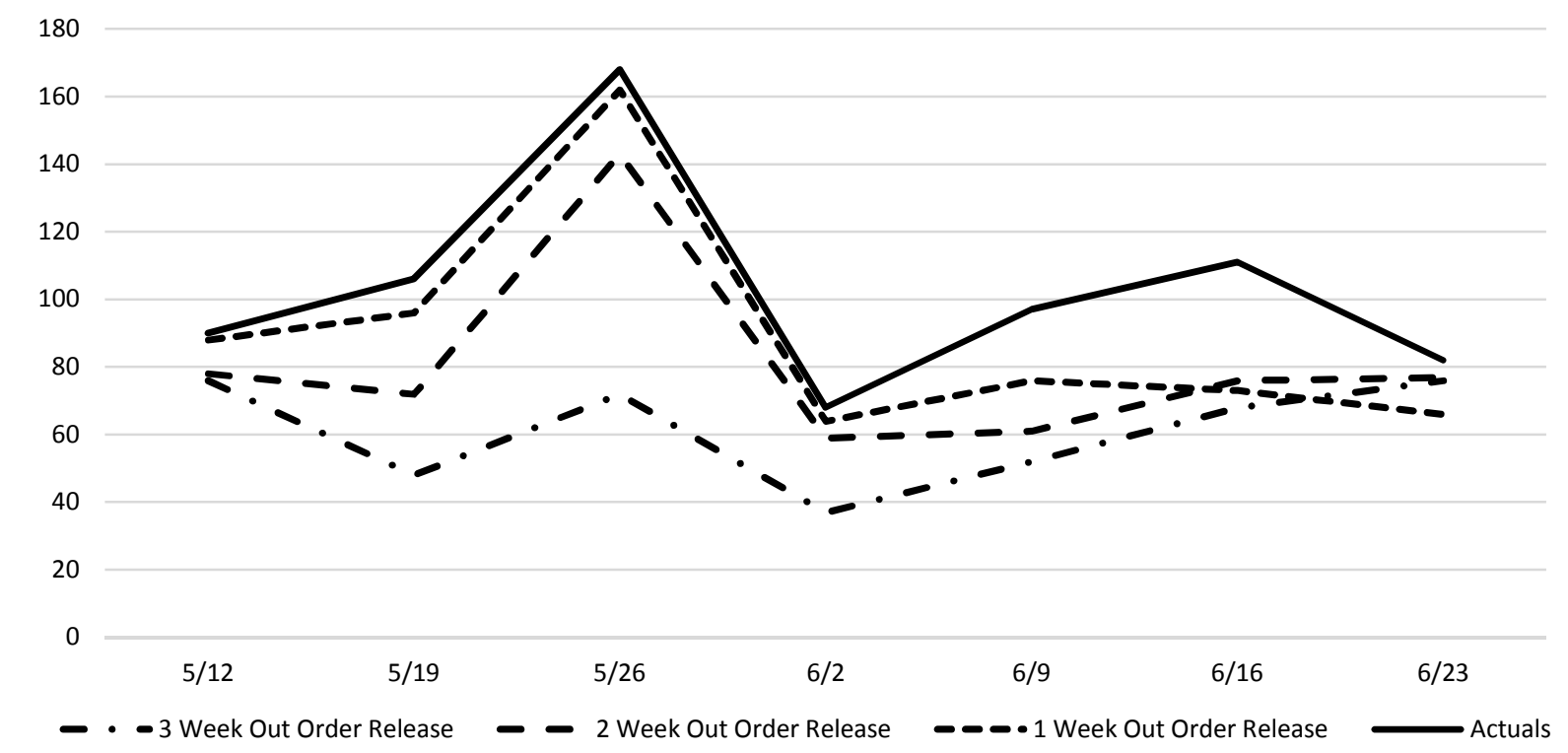

Fig. 1. Sample observed data received in order releases by Parts Inc. for a single part number.

These data trends may be useful for creating better production plans for future periods so that total cost can be minimized. We investigated two approaches to achieve this. The first is an optimization method, which is somewhat similar to the model of Graves et al. [5], in logic. This 
method uses not only the actual demand data, but also the order release evolution for each actual demand for a number of weeks. This means, it implicitly uses the order release percentage change information as well. Thus, all three data trends are utilized in this approach.

The second approach is based on an observation of the bias in order release changes and adjusting for that. Next we discuss these methods.

\section{OPTIMIZATION MODEL}

The basic idea of the optimization model is similar to the Graves et al. [5] single stage model. That is, the primary goal is to find a way to convert the incoming new order release information into a revision in the production plan. Both models use order release information for the next $\mathrm{H}$ periods. In our model, we use actual order release information and make no assumptions regarding the data, whereas Graves et al. [5] use a stochastic model for this order release process and assume that customer forecasts are unbiased, they improve as they are revised and the customer forecast error over the horizon matches the variability in the demand process. Since our model makes no assumptions, there is no need to perform a check and convert the data to confirm those assumptions before applying the model. This significantly improves the practicality of our model.

In both models, a key constraint is related to the total change in order releases. Graves et al. [5] equates this change to the total change in the production plan. That is, they require that the cumulative revision to the production plan must equal the cumulative order release revision in each period. In our model, we relax this constraint and use the cumulative order release revision as an upper bound on the cumulative revision to the production plan in each period. This provides more flexibility to the model.

Another key component of the models is how the production plan for a week is calculated. In 
both models, this is done through a linear system, where a weighted sum of order release revisions is used to decide on the change that will be made to the production plan.

The objective of our model is to minimize the cost of shortage and overage, whereas Graves et al. [5] focus on minimizing the variance in production plans. With these key components, our optimization model is as follows:

\section{Data}

$N$ : Data horizon for calculating optimal weights.

$H:$ Order release horizon. At each time period $1 \leq t \leq N$

an order release for the requirements for the next H periods.

$f_{t}(t+i)=$ Order release for time $t+i$ at time $t, \quad i=1,2, \ldots H$

$f_{t}(t)=$ Actual order quantity at time $t$

$\Delta f_{t}(t+i)=f_{t}(t+i)-f_{t-1}(t+i) \quad$ (Order release rivison for time $t+i$ at time $t, i=$ $1,2, \ldots H)$

$p=$ unit shortage cost

$h=$ unit overage cost

\section{Decision Variables}

$F_{t}(t+i)=$ Production plan for time $t+i$ at time $t, \quad i=1,2, \ldots H$

$F_{t}(t)=$ Actual production completed at time $t$

$\Delta F_{t}(t+i)=F_{t}(t+i)-F_{t-1}(t+i) \quad$ (Production plan revision for time $t+i$ at time $t$, $i=1,2, \ldots H)$

$w_{i j}=$ Proportion of the order release change for period $t+j$ added to the production plan for period $t+i$

\section{Model}




$$
\begin{array}{lc}
\text { minimize } p \sum_{t=1}^{N}\left(f_{t}(t)-F_{t}(t)\right)^{+}+h \sum_{t=1}^{N}\left(F_{t}(t)-f_{t}(t)\right)^{+} \\
\Delta F_{t}(t+i)=\sum_{j=0}^{H} w_{i j} \Delta f_{t}(t+j) & i=0,1, \ldots H \\
\sum_{i=0}^{H} \Delta F_{t}(t+i) \leq \sum_{j=0}^{H} \Delta f_{t}(t+j) & t=1,2, \ldots N \\
\sum_{i=0}^{H} w_{i j} \leq 1 & j=0,1, \ldots H \\
0 \leq w_{i j} \leq 1 & i, j=0,1, \ldots H
\end{array}
$$

In this model, (1) is the objective function that minimizes the cost of shortage and overage. (2) calculates the change that will be made to the production plan for a time period $t+i$ as a weighted sum of forecast revisions. (3) sets the cumulative order release revision as an upper bound on the cumulative revision to the production plan in each time period. (4) limits the sum of weights, which are the proportion of the order release revisions for $t+j$ that is added to the production plan for $t+i$.

\section{HEURISTIC APPROACHES}

As mentioned earlier, in our study we use real data from the automotive industry and make no assumptions about the incoming order releases to Parts Inc. One of the findings of Altintas and Trick [7], who analyzed the order release behavior of automotive manufacturers, is that OEMs tend to be biased, i.e. most of them tend to increase or decrease their forecast consistently. In this study we find that Car Co.'s order releases are also biased for most of the parts. Fig. 2 illustrates the percentage changes in 1 week-out order releases for a period of more than a year. Since there are outliers, looking at the median percentage change is more robust than the mean percentage change. For this part the median percentage change is $7.11 \%$. We looked at many other parts and 
found similar results. This clearly shows us that the 1 week-out order releases are biased and Car Co. consistently changes its actual order quantity at the last minute. Using this observation, we tested adjusting the 1-week out order releases using two methods: In the first approach, we find the optimal adjustment factor solving a simple optimization model as follows.

\section{Data}

N: Data horizon for calculating the optimal adjustment factor.

$f_{t-1}(t)=$ Order release for time $t$ at time $t-1$

$f_{t}(t)=$ Actual order quantity at time $t$

$p=$ unit shortage cost

$h=$ unit overage cost

Decision Variables

$A D J=$ Adjustment factor

$F_{t}(t)=$ Production plan for time $t+1$ at time $t$

\section{Model}

minimize $p \sum_{t=1}^{N}\left(f_{t}(t)-F_{t}(t)\right)^{+}+h \sum_{t=1}^{N}\left(F_{t}(t)-f_{t}(t)\right)^{+}$

$F_{t}(t)=A D J * f_{t-1}(t) \quad t=1, \ldots N$

In this model, (6) is the objective function that minimizes the cost of shortage and overage, which is exactly the same as (1). (7) calculates the production plan for time period $t$ using the order release quantity and the adjustment factor.

In the second approach, we use the historical median percentage change and use it as the production plan. 


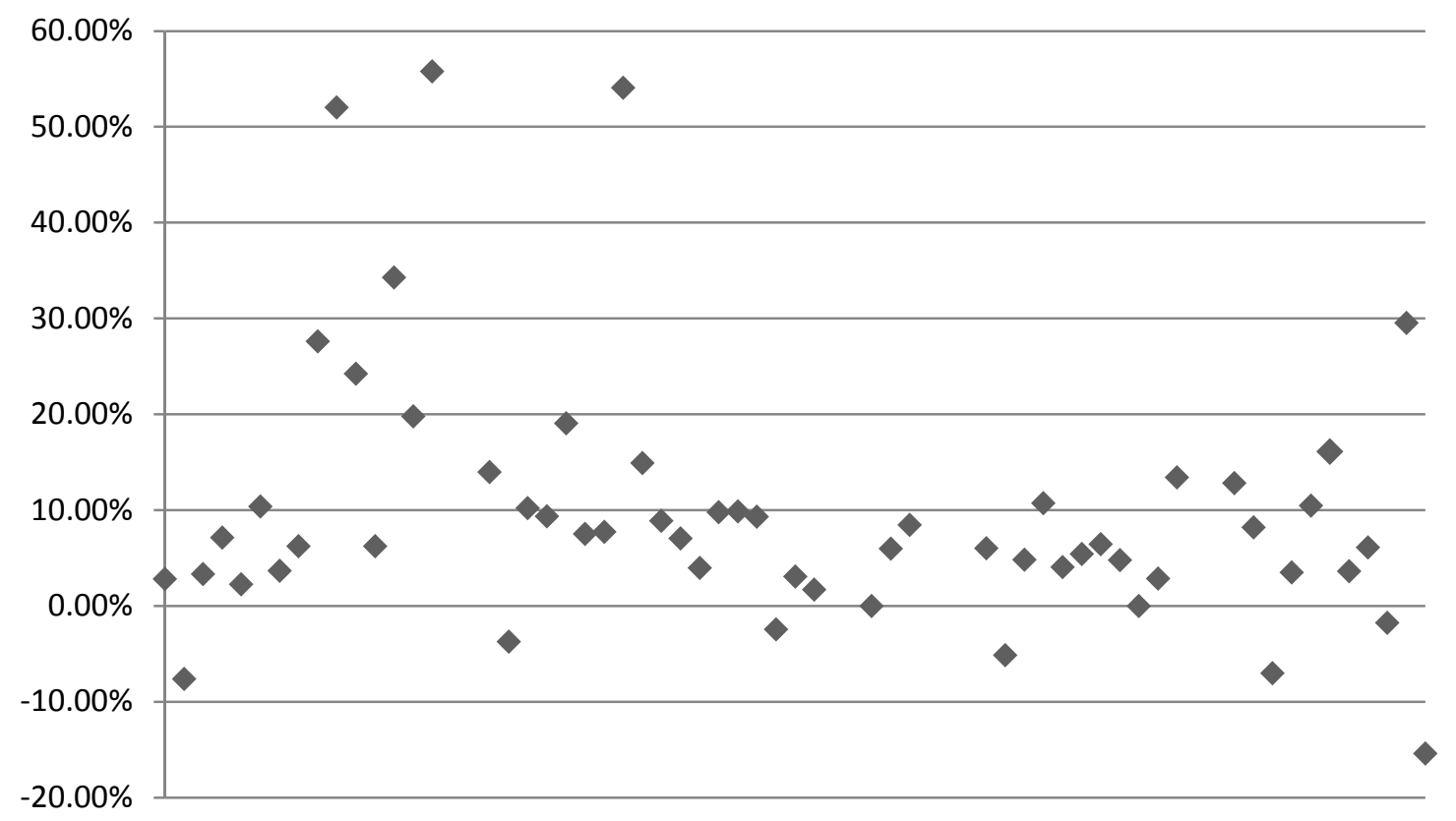

Fig. 2. Sample observed data of percentage changes from 1 week out order release to actual orders for a single part number.

Similar to our optimization model presented in Section IV, this heuristic, regardless of the way the adjustment factor is calculated, also makes no assumptions regarding the data. So there is no need to perform a check and convert the data to confirm those assumptions before applying the heuristic. Furthermore, the adjustment factors can be automatically updated as new data is received over time.

In addition, one of the biggest advantages of the median adjustment heuristic approach is its robustness and ease of use. As it is the case of any real data set, the data Parts Inc. receives from Car Co. has various irregularities. Since we only need to calculate the median adjustment factor, there is no need for data cleaning and preparation, thus the process can be automated easily. Furthermore, everything can be done in a spreadsheet environment with the use of advanced formulas that do not need any manual intervention once setup. 


\section{RESULTS}

In this section, we provide results for five parts that resulted in excessive premium freight costs for the Parts Inc. Both methods are implemented in Microsoft Excel. The optimization models utilize OpenSolver [17]. In our tests, for the optimization method, we used actual demand for each week along with the customer forecasts for the next five weeks. Thus $\mathrm{H}=6$ in our optimization model. For both methods, we use several weeks of data to calculate the optimal weights and the adjustment factors, respectively. Then, we use the values to test the performance of the methods and validate these methods on another set of data for the same part. In Table II, OPT refers to the results for the optimization model. OR refers to the results of the order release being used with no modifications. HEURISTIC-OPT refers to the results for the optimization based adjustment heuristic. HEURISTIC-MEDIAN refers to the results for the median adjustment heuristic. The metrics of interest are total cost, shortage percentage, average shortage quantity and average overage quantity. Cost consists of both shortage and overage costs. Consistent with the focus on premium freight shipments, the per unit shortage cost is assumed to be twice as much as the per unit overage cost. Shortage percentage provides the frequency of shortage occurrence. The average shortage and overage quantities provide the magnitude of shortages and overages, respectively. 
Overall, the simple median adjustment heuristic performs best. The optimization based heuristic adjustment performs second. The optimization model performs third best. Relying

TABLE II

PERFORMANCE COMPARISON OF METHODOLOGIES BY PART

\begin{tabular}{|c|c|c|c|c|c|}
\hline Parts & Part 1 & Part 2 & Part 3 & Part 4 & Part 5 \\
\hline Cost (OR) & 395.0 & 327.0 & 1018.0 & 304.0 & 347.0 \\
\hline Cost (OPT) & 534.7 & 214.0 & 794.0 & 523.5 & 204.2 \\
\hline Cost (HEURISTIC-OPT) & 341.0 & 194.0 & 503.9 & 237.0 & 184.5 \\
\hline Cost (HEURISTIC-MEDIAN) & 228.4 & 181.3 & 278.1 & 184.5 & 177.9 \\
\hline Shortage \% (OR) & $90.0 \%$ & $83.3 \%$ & $95.7 \%$ & $80.0 \%$ & $82.6 \%$ \\
\hline Shortage \% (OPT) & $40.0 \%$ & $52.4 \%$ & $55.0 \%$ & $50.0 \%$ & $57.1 \%$ \\
\hline Shortage \% (HEURISTIC-OPT) & $10.0 \%$ & $21.4 \%$ & $0.0 \%$ & $33.3 \%$ & $32.0 \%$ \\
\hline Shortage \%(HEURISTIC-MEDIAN) & $35.0 \%$ & $29.2 \%$ & $43.5 \%$ & $35.0 \%$ & $26.1 \%$ \\
\hline Avg. Shortage Qty (OR) & 9.6 & 7.3 & 25.3 & 7.2 & 7.8 \\
\hline Avg. Shortage Qty (OPT) & 8.6 & 3.6 & 14.0 & 10.0 & 3.4 \\
\hline $\begin{array}{c}\text { Avg. Shortage Qty (HEURISTIC- } \\
\text { OPT) }\end{array}$ & 2.2 & 1.1 & 0.0 & 0.5 & 1.3 \\
\hline $\begin{array}{c}\text { Avg. Shortage Qty (HEURISTIC- } \\
\text { MEDIAN) }\end{array}$ & 3.3 & 1.3 & 3.0 & 1.4 & 1.5 \\
\hline Avg. Overage Qty (OR) & 0.7 & 1.0 & 0.4 & 2.4 & 1.0 \\
\hline Avg. Overage Qty (OPT) & 9.6 & 2.9 & 11.6 & 9.0 & 3.0 \\
\hline $\begin{array}{c}\text { Avg. Overage Qty (HEURISTIC- } \\
\text { OPT) }\end{array}$ & 12.7 & 7.1 & 25.2 & 12.1 & 6.2 \\
\hline $\begin{array}{c}\text { Avg. Overage Qty (HEURISTIC- } \\
\text { MEDIAN) }\end{array}$ & 4.8 & 6.0 & 7.9 & 7.5 & 5.4 \\
\hline
\end{tabular}

solely on the order releases usually results in the worst outcomes. More specifically, in terms of cost, both adjustment heuristics resulted in significantly lower costs. The optimization model, on the other hand performs fairly well compared to relying on order releases. For three out of five parts, the optimization model is able to obtain lower costs than relying solely on order releases. 
Since the shortages result in overtime production and expedited premium freight shipments, the frequency and magnitude of shortages is very important. Again, both of the adjustment heuristics perform best for all five parts. Optimization model also performs very well compared to relying on the order releases. One downside is the increase in the overages for both methods. These overages are excess production and inventory that is used in the next week's production.

The five parts that are presented in Table II are all produced in the same plant of Parts Inc. To see if the results hold in other parts' data, we applied both methods on another set of five parts from a different plant of Parts Inc. The results are presented in Table III, which are similar to those of Table II, in general. One difference this time is the fact that, for parts 9 and 10 the bias was actually small and positive (i.e. the actual orders are less than the 1-week out customer forecasts). Even though the focus and motivation of this study is the set of parts that results in high expedited shipping costs due to shortage, we have included these parts for illustrative purposes. For parts 9 and 10, the median based adjustment heuristic happens to perform better than the order release in terms of cost, but the difference is not very significant. In general, the heuristic adjustment may not perform better than solely relying on order releases for such parts with positive bias. Because the bias correction potentially can cause higher shortage costs since it has a higher weight in our cost objective function. We also see the impact of decreasing the order quantity as an increase in the frequency of shortages and the average shortage quantity for these two parts. For the parts with negative bias (Parts 6-8), the median based adjustment heuristic performs best in terms of cost and shortages, which is similar to the results for Parts 15. The optimization based adjustment heuristic performs second best for three of the five parts. The optimization method, on the other hand, performs third best for two of the five parts, but for the remaining three parts, relying on order release information performs better than the 
optimization method. Interestingly, optimization method results in the least cost among the four methods for part 9, which has a positive bias.

TABLE III

Performance Comparison of Methodologies by PART

\begin{tabular}{|c|c|c|c|c|c|}
\hline Parts & Part 6 & Part 7 & Part 8 & Part 9 & Part 10 \\
\hline Cost (OR) & 340.0 & 2781.0 & 58.0 & 1357.0 & 329.0 \\
\hline Cost (OPT) & 594.0 & 1936.5 & 161.9 & 1257.4 & 359.2 \\
\hline Cost (HEURISTIC-OPT) & 300.9 & 1373.4 & 64.4 & 1346.4 & 339.4 \\
\hline Cost (HEURISTIC-MEDIAN) & 310.2 & 1372.7 & 54.1 & 1346.5 & 305.2 \\
\hline Shortage \% (OR) & $44.4 \%$ & $93.8 \%$ & $50.0 \%$ & $25.0 \%$ & $12.5 \%$ \\
\hline Shortage \% (OPT) & $66.7 \%$ & $50.0 \%$ & $56.3 \%$ & $43.8 \%$ & $18.8 \%$ \\
\hline Shortage \% (HEURISTIC-OPT) & $27.8 \%$ & $43.8 \%$ & $12.5 \%$ & $25.0 \%$ & $12.5 \%$ \\
\hline $\begin{array}{c}\text { Shortage \%(HEURISTIC- } \\
\text { MEDIAN) }\end{array}$ & $38.9 \%$ & $43.8 \%$ & $18.8 \%$ & $37.5 \%$ & $18.8 \%$ \\
\hline Avg. Shortage Qty (OR) & 6.7 & 83.8 & 1.2 & 16.3 & 3.8 \\
\hline Avg. Shortage Qty (OPT) & 14.8 & 42.2 & 4.0 & 19.2 & 4.3 \\
\hline $\begin{array}{c}\text { Avg. Shortage Qty (HEURISTIC- } \\
\text { OPT) }\end{array}$ & 3.8 & 26.8 & 0.3 & 16.7 & 3.7 \\
\hline $\begin{array}{c}\text { Avg. Shortage Qty (HEURISTIC- } \\
\text { MEDIAN) }\end{array}$ & 5.0 & 26.9 & 0.5 & 20.8 & 4.2 \\
\hline Avg. Overage Qty (OR) & 5.4 & 6.2 & 1.3 & 52.3 & 12.9 \\
\hline Avg. Overage Qty (OPT) & 3.4 & 36.7 & 2.2 & 40.3 & 13.9 \\
\hline $\begin{array}{c}\text { Avg. Overage Qty (HEURISTIC- } \\
\text { OPT) }\end{array}$ & 9.1 & 32.2 & 3.4 & 50.7 & 13.8 \\
\hline $\begin{array}{c}\text { Avg. Overage Qty (HEURISTIC- } \\
\text { MEDIAN) }\end{array}$ & 7.3 & 32.0 & 2.4 & 42.5 & 10.8 \\
\hline
\end{tabular}

When we compare the two adjustment heuristics, we find a somewhat surprising result.

Overall, the simple median adjustment approach performs better than the optimization based adjustment in terms of total cost. It seems that the optimization based adjustment suffers from overfitting as it generally results in a much higher adjustment factor that performs very well for 
the training data but does not perform as well in the test data. For five of the eight parts with negative bias, the adjustment factor calculated by the optimization model is at least $50 \%$ more than what the median based adjustment suggests. Because of this, the optimization based adjustment results is lower shortage percentage and quantity, and unnecessarily high average overage quantity. On the other hand, for the remaining two parts with positive bias, the optimization based adjustment factor significantly underestimates the positive bias. Because of that, the same higher overage quantity and lower shortage percentage and quantity are observed for these parts as well. This overall difference between the two adjustment approaches also gives us an indication of the less than expected performance of the optimization method as that method suffers from overfitting to the training data.

One other interesting result emanates from the analysis of the weights obtained from the optimization model as illustrated in Table IV for two parts produced by Parts Inc.

In these tables, the first column is the proportion of the order release change for the current week added to the next 4 weeks' production plans. This is somewhat similar to the bias adjustment done in the median adjustment heuristic. Interestingly, the summation of these weights is very high even though the information is past. Also, these weights do not seem to have a pattern.

The diagonal cells shaded in grey are production plan revision weights for target production week, based on order release adjustment for that week. Similar to what Graves et al (1998) found, in a column, weights always peak at these diagonal cells and the weights usually drop exponentially around the diagonal. This can be interpreted as the change of order release information is most relevant for the matching week that's planned for. The cells in the upper right side of the diagonal are adjustment weights based on predicted future changes that occur 
following the target week. In contrast, the cells below and left of the diagonal are adjustment weights based on predicted future changes that occur prior to the target week.

TABLE IV

OBSERVED OPTIMIZED WEIGHTS FOR ORDER RELEASE CHANGE REDISTRIBUTION

\begin{tabular}{|c||c|c|c|c|c|}
\hline Part 2 & $\mathrm{T}+0$ & $\mathrm{~T}+1$ & $\mathrm{~T}+2$ & $\mathrm{~T}+3$ & $\mathrm{~T}+4$ \\
\hline $\mathrm{T}+0$ & 0.14 & $\mathbf{0 . 7 6}$ & 0.12 & 0.00 & 0.00 \\
\hline+1 & 0.09 & 0.17 & $\mathbf{0 . 8 8}$ & 0.00 & 0.00 \\
\hline $\mathrm{T}+2$ & 0.22 & 0.03 & 0.00 & 1.00 & 0.00 \\
\hline $\mathrm{T}+3$ & 0.00 & 0.00 & 0.00 & 0.00 & $\mathbf{0 . 9 8}$ \\
\hline $\mathrm{T}+4$ & 0.38 & 0.04 & 0.00 & 0.00 & 0.02 \\
\hline Sum & 0.83 & 1.00 & 1.00 & 1.00 & 1.00 \\
\hline
\end{tabular}

\begin{tabular}{|c||c|c|c|c|c|}
\hline Part 3 & $T+0$ & $T+1$ & $T+2$ & $T+3$ & $T+4$ \\
\hline$T+0$ & 0.64 & 1.00 & 0.00 & 0.00 & 0.00 \\
\hline$T+1$ & 0.00 & 0.00 & $\mathbf{0 . 9 2}$ & 0.06 & 0.08 \\
\hline$T+2$ & 0.08 & 0.00 & 0.00 & $\mathbf{0 . 9 2}$ & 0.00 \\
\hline$T+3$ & 0.22 & 0.00 & 0.00 & 0.01 & $\mathbf{0 . 7 9}$ \\
\hline$T+4$ & 0.06 & 0.00 & 0.08 & 0.00 & 0.02 \\
\hline Sum & 1.00 & 1.00 & 1.00 & 1.00 & 0.89 \\
\hline
\end{tabular}

We see that the column summations (last rows) always equal to 1 except for the first column. This means the all the order release change information is used in revising the production plans. All of these observations hold true across all ten parts investigated.

\section{CONCLUSION}

In the automotive industry, OEMs share their order releases, for multiple periods with their suppliers and update these order releases frequently before the due date. Suppliers also update their production plans and pass these updates to upstream suppliers. As largely assumed in the literature, assuming that these customer forecasts may provide valuable information, we develop 
a model that takes the customer forecast updates as input and converts this into a production plan. This model is similar to the single stage model developed by Graves et al. [5]. The model provides mixed results. For some parts, it is able to generate a production plan better than the order releases received. In some other parts, it performs worse than just relying on the order release received. Although the performance of the model is not great as a forecasting tool, the weights generated provide some useful insights similar to what Graves et al. [5] have found, without making any of the assumptions regarding the structure of the data. Thus, our findings confirm and extend their findings.

Because of the way managers make forecasts, such as a myopic behavior, customer forecast adjustments and updates are not necessarily information-adding in all scenarios. Moreover, in the JIT setting we consider, there is a strong emphasis on avoiding inventories in ordering amounts placed by Car Co., which may be one of the reasons for the systematic, mostly negative, bias in the order quantities that are being placed by Car Co. The JIT emphasis may be causing the order release quantities to be lower than the actual demand that occurs in order to avoid having excess inventory within the supply chain.

We find that when there is bias in the order release updates, it is likely that the information can contain so little use (because of their randomness) and cause significant disruptions (potentially wrong or poor planning) in the supply chain if exchanged and acted upon. To remedy this, we develop two versions of a simple adjustment heuristic to adjust the order release information to create production plans. Although the optimization based adjustment heuristic was expected to perform best, surprisingly, the simpler median based adjustment heuristic performs best. This may be attributed to overfitting of the adjustment factor in the former approach. The median adjustment heuristics performs very well especially when the bias is negative and significant. 
When the bias is positive, the heuristic does not perform well since the emphasis is on shortage costs compared to overage cost. When there is no bias, the heuristic makes no adjustment to the order release information, thus it has no impact. Part of the reason we explored using a heuristic was based on the reaction we had from Parts Inc. when discussing different forecasting approaches and optimization methodologies. The primary concerns expressed by the management during interviews were not understanding the methodologies and the challenge of maintaining a complex methodology without adequate support and with possible turnover of employees, who are familiar with such a complex methodology.

A major benefit of being able to adjust order release information to better production plans is providing increased visibility to the upstream supply chain. Incorporating the adjusted releases in the information communicated to the suppliers provides (some) forward visibility. It is known that information visibility can reduce bullwhip effect. The median based adjustment that we are suggesting is easy to implement in practice and outperforms both the optimization based adjustment heuristic and the optimization method in general.

One limitation of this paper is its reliance on a single firm's data. It would be very useful to test the proposed methods on data from other firms to investigate if similar results can be obtained.

\section{REFERENCES}

[1] Lee, H. L., Padmanabhan, V., \& Whang, S. "Information distortion in a supply chain: the bullwhip effect." Management Science, vol. 43, no. 4, pp. 546-558, Apr. 2004.

[2] Lee, H. L., So, K. C., \& Tang, C. S. "The value of information sharing in a two-level supply chain.” Management science, vol. 46, no. 5, pp. 626-643, May 2000. 
[3] Chen, H., Chen, J., \& Chen, Y. F. “A coordination mechanism for a supply chain with demand information updating." International Journal of Production Economics, vol. 103, no. 1, pp. 347-361, Sep. 2006.

[4] Cattani, K., \& Hausman, W. "Why are forecast updates often disappointing?” Manufacturing \& Service Operations Management, vol 2, no. 2, pp. 119-127. Spring 2000.

[5] Graves, S. C., Kletter, D. B., \& Hetzel, W. B. “A dynamic model for requirements planning with application to supply chain optimization." Operations Research, vol. 46 no. 3, S35-S49. May-Jun 1998

[6] Huggins, E. L., \& Olsen, T. L. "Supply chain management with guaranteed delivery”. Management Science, vol. 49, no. 9, pp. 1154-1167. Sep. 2003.

[7] Altintas, N., \& Trick, M. “A data mining approach to forecast behavior.” Annals of Operations Research, vol. 49, no. 1, pp. 3-22. Nov. 2012.

[8] Hausman, W. H. "Sequential decision problems: A model to exploit existing forecasters." Management Science, vol.6, no. 2, pp. B93-B111, Oct. 1969.

[9] Baker, K. "Requirements Planning," in Handbooks in Operations Research and Management Science, Logistics of Production and Inventory. Vol 4. Amsterdam, Netherlands. NorthHolland, 1993.

[10] Sarimveis, H., Patrinos, P., Tarantilis, C. D., \& Kiranoudis, C. T. "Dynamic modeling and control of supply chain systems: A review." Computers \& Operations Research, vol. 35, no. 11, pp. 3530-3561. Nov. 2008.

[11] Heath, D. C., \& Jackson, P. L. "Modeling the evolution of demand forecasts ITH application to safety stock analysis in production/distribution systems." IIE transactions, vol. 26, no. 3, pp. 17-30. May 1994. 
[12] Graves, S. C., \& Willems, S. P. “Optimizing strategic safety stock placement in supply chains." Manufacturing \& Service Operations Management, vol. 2, no. 1, pp. 68-83. Jan. 2000.

[13]Aviv, Y. "The effect of collaborative forecasting on supply chain performance." Management science, vol. 47, no. 10, pp. 1326-1343. Oct. 2001.

[14]Aviv, Y. "On the benefits of collaborative forecasting partnerships between retailers and manufacturers." Management Science, vol 53, no. 5, pp. 777-794. May 2007.

[15]Terwiesch, C., Ren, Z. J., Ho, T. H., \& Cohen, M. A. “An empirical analysis of forecast sharing in the semiconductor equipment supply chain.” Management Science, vol. 51, no. 2, pp. 208-220. Feb. 2005.

[17] Mason, A.J., "OpenSolver - An Open Source Add-in to Solve Linear and Integer Progammes in Excel", Operations Research Proceedings 2011, eds. Klatte, Diethard, Lüthi, Hans-Jakob, Schmedders, Karl, Springer Berlin Heidelberg pp 401-406, 2012, http://dx.doi.org/10.1007/978-3-642-29210-1_64, http://opensolver.org

[19] Armstrong, J. S., Lusk, E. J., Gardner Jr, E. S. , Geurts, M. D., Lopes, L. L., Markland, R. E., McLaughlin, R. L., Newbold, P., Pack, D. J., Andersen, A., Carbone, R., Fildes, R., Parzen, E., Newton, H. J., Winkler, R. L., \& Makridakis, S.S.S. "Commentary on the Makridakis Time Series Competition (M-Competition)," Journal of Forecasting, vol. 2, no. 3, pp. 259-311, 1983.

[20] Ho, W., Zheng, T., Yildiz, H., \& Talluri, S. Supply chain risk management: a literature review. International Journal of Production Research, 53(16), 5031-5069, 2015. 
APPENDICES

Appendix 1: Sample full raw data with 40-week out order releases for a specific part.

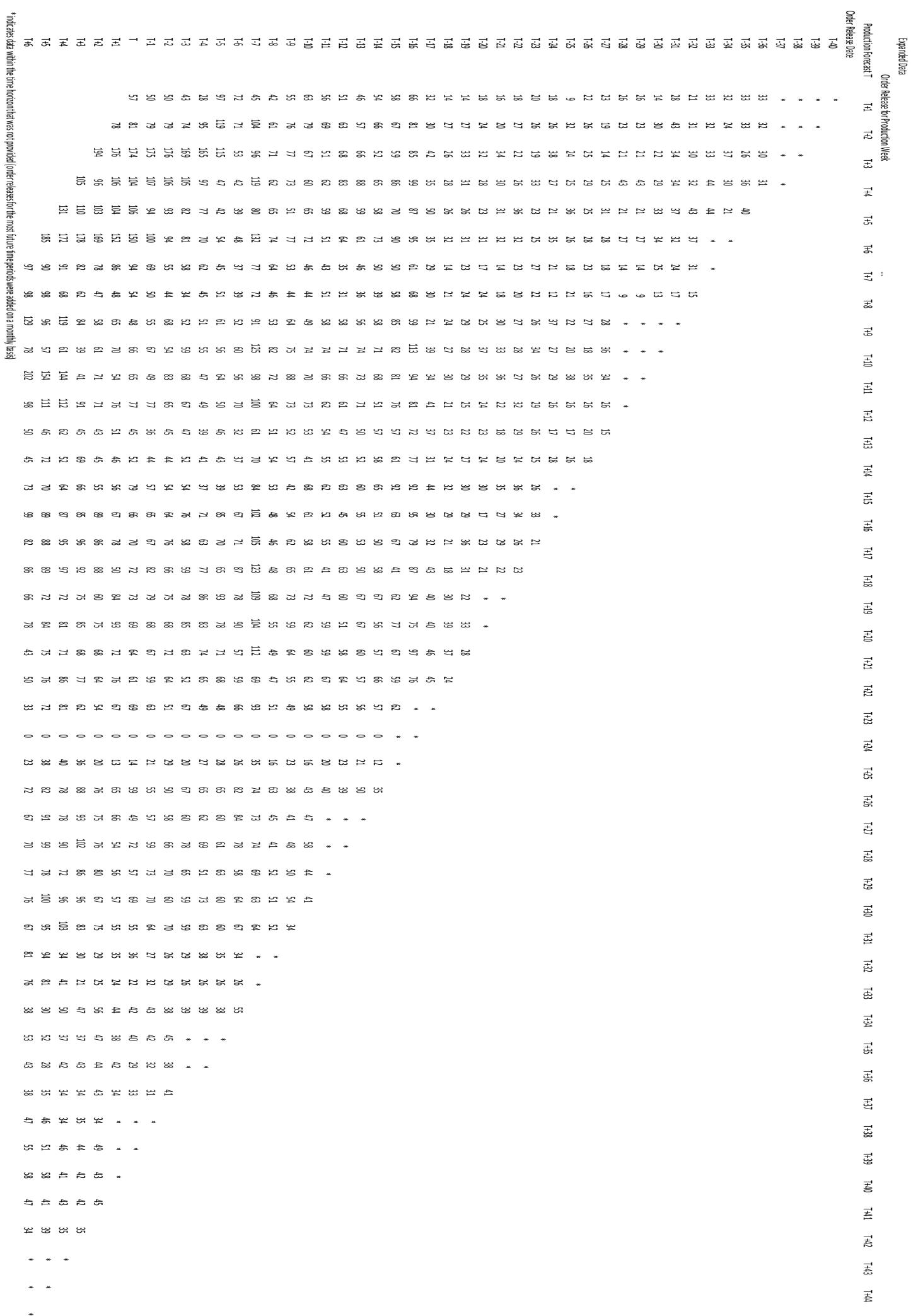

